\title{
Focusing of Micrometer-Sized Metal Particles Enabled by Reduced Acoustic Streaming via Acoustic Forces in a Round Glass Capillary
}

\author{
M. S. Gerltø, ${ }^{1,{ }^{*}}$ A. Paeckel, ${ }^{1}$ A. Pavlic $\odot,{ }^{1}$ P. Rohner $\odot,{ }^{2}$ D. Poulikakos $\odot,{ }^{2}$ and J. Dual ${ }^{1}$ \\ ${ }^{1}$ Mechanics and Experimental Dynamics, Department of Mechanical and Process Engineering, ETH Zurich, 8092 \\ Zurich, Switzerland \\ ${ }^{2}$ Laboratory of Thermodynamics in Emerging Technologies, Department of Mechanical and Process Engineering, \\ ETH Zurich, 8092 Zurich, Switzerland
}

(Received 26 May 2021; revised 10 September 2021; accepted 6 December 2021; published 31 January 2022)

\begin{abstract}
Two-dimensional (2D) metal-particle focusing is an essential task for various fabrication processes. While acoustofluidic devices can manipulate particles in two-dimensions, the production of these devices often demands a cleanroom environment. Therefore, acoustically excited glass capillaries present a cheap alternative to labor-intensive cleanroom production. Here, we present 2D metal microparticle focusing in a round glass capillary using bulk acoustic waves. Excitation of the piezoelectric transducer at specific frequencies leads to mode shapes in the round capillary, concentrating particles toward the capillary center. We experimentally investigate the particle line width for different particle materials and concentrations. We demonstrate the focus of copper particles approximately $1 \mu \mathrm{m}$ in diameter down to a line of width $60.8 \pm 7.0 \mu \mathrm{m}$ and height $45.2 \pm 9.3 \mu \mathrm{m}$, corresponding to a local concentration of $4.5 \% \mathrm{v} / \mathrm{v}$, which is 90 times higher than the concentration of the initial solution. Further, we achieve the focusing of $1-\mu \mathrm{m}$ polystyrene particles, which is usually prevented due to acoustic streaming. Through numerical analysis, we reveal the mechanism enabling the manipulation of particles in the low-micrometer range. Due to a transition of the acoustic streaming patterns from two dominant vortices in the lower half to two dominant vortices in the upper half, the streaming velocity exhibits a local minimum while the overall acoustic energy density stays at a sufficiently high level for particle focusing, leading to a lower critical particle radius than in conventional rectangular microchannels. Finally, we use our method to eject copper particles through a tapered round capillary with an opening of $25 \mu \mathrm{m}$ in diameter, which would not be possible without particle focusing. Our setup can be utilized for various applications that otherwise might suffer from abrasion, clogging, and limited resolution.
\end{abstract}

DOI: 10.1103/PhysRevApplied.17.014043

\section{INTRODUCTION}

Acoustophoresis, the manipulation of particles utilizing acoustic forces, is one of the most popular techniques for particle manipulation because it is noninvasive, label free, and biocompatible [1]. To a great extent, acoustofluidic devices are based on microchannels fabricated in silicon, glass, or polydimethylsiloxane (PDMS) [2]. Despite the flexibility in design, the production of microchannels in glass and silicon relies on expensive and labor-intensive cleanroom procedures and, depending on the resolution, PDMS microchannels require silicon stamps produced in

\footnotetext{
*mgerlt@ethz.ch
}

Published by the American Physical Society under the terms of the Creative Commons Attribution 4.0 International license. Further distribution of this work must maintain attribution to the author(s) and the published article's title, journal citation, and DOI. a cleanroom environment [3,4]. Further, acoustic particle manipulation inside PDMS cavities usually requires interdigital transducers (IDTs), which need to be designed carefully and evaporated with high-end equipment.

An approach involving less expensive equipment and a reduction of manual labor is the application of glass capillaries in combination with bulk acoustic waves. Here, a piezoelectric element (piezo) is glued to an off-the-shelf glass capillary. On excitation of the piezo with an ac signal, the capillary can vibrate in specific modes that lead to beneficial acoustic potentials inside it. This procedure has been used for various applications such as biomedical analysis [5], blood trapping [6], seed-particle trapping for sample washing [7], nanoparticle enrichment [8], and two-dimensional (2D) concentration of microparticles [9]. Additionally, intensive numerical investigations have been carried out concerning acoustic particle manipulation inside glass capillaries [10-12].

Most of the aforementioned works utilize capillaries with rectangular cross sections and are interested in 
particle trapping. Here, we show 2D particle focusing in a glass capillary with a round cross section, as also demonstrated by Goddard et al. $[13,14]$. We present the 2D focusing of metal particles that are close to the theoretical radiation force-driven manipulation size limit of acoustofluidics in common rectangular microfluidic channels (the critical particle radius $r_{c}$ ) [15]. In contrast to the literature, we utilize a pair of piezoelectric elements (piezos) to improve the focusing performance while remaining at a low input voltage of approximately $15 \mathrm{~V}_{\mathrm{PP}}$ (Peak to Peak), which enables operation without a power amplifier. With a selfwritten MATLAB code and a microscope setup that allows us to inspect the top and side views of the capillary simultaneously, we characterize the performance of our device by analyzing the particle line width for different particle sizes, materials, flow rates, and concentrations. Our characterization reveals that the critical particle radius for polystyrene (PS) particles is lower in our system than in conventional rectangular microchannels. We are able to explain this performance through numerical investigations, showing a local minimum in the average streaming velocity, while maintaining a high acoustic radiation force close to our experimentally verified focusing frequency. The minimization of acoustic streaming has been thoroughly investigated in the literature and has been achieved by various approaches such as pulsed excitation [16], inhomogeneous fluids [17], and shape-optimized channels [18]. The latter work suggests a curved shape for optimal streaming suppression, which supports our findings. Furthermore, since analytical solutions for a curved system are not available, we numerically evaluate the critical particle radius and find a value of $0.35 \mu \mathrm{m}$ for PS particles at the frequency with the minimal streaming velocity, further supporting our findings. However, we would like to mention that the approximations of the critical particle radius, derived by balancing the acoustic radiation force and drag from acoustic streaming, only lead to first estimates, which do not accurately describe the performance of an individual device due to the spatially dependent relation between the two forces [19].

Finally, we employ our setup for the stable ejection of highly concentrated metal particles using a nozzle with an opening diameter of $25 \mu \mathrm{m}$. Given that the aperture of the nozzle is only approximately 23 times bigger than the particle diameter, continuous ejection without clogging would not be feasible without sufficient particle focusing.

Our investigations and setup are relevant for a wide variety of industrial applications that rely on particle ejection out of small nozzles, including, e.g., metal threedimensional (3D) printing and water-jet cutting.

\section{OPERATING PRINCIPLE}

The acoustofluidic device consists of a round glass capillary with two piezoelectric elements (piezos) attached

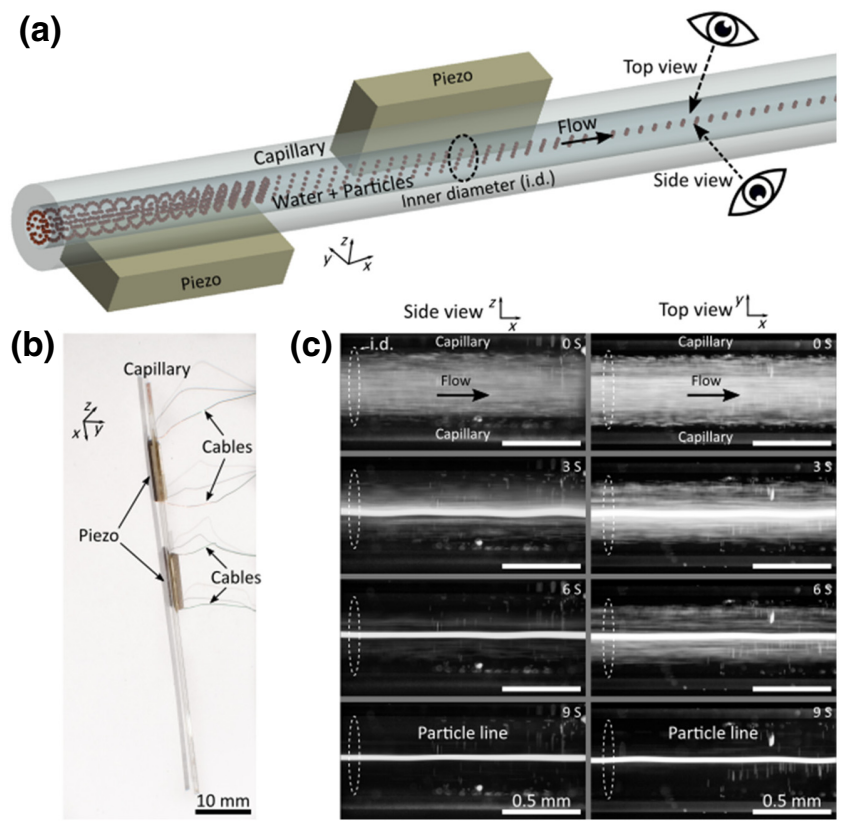

FIG. 1. The design and working principle of 2D particle focusing in a round glass capillary. (a) A sketch of the design. (b) A photograph of the acoustofluidic device. (c) A micrograph series of $2 \mathrm{D}$ focusing with 5 - $\mu \mathrm{m}$-diameter fluorescent polystyrene particles from the side and top view. The photographs are taken from a location $4 \mathrm{~mm}$ away from where the particles exit the glass capillary. The particles are pumped through the device at a flow rate of $100 \mu 1 \mathrm{~min}^{-1}$. The piezoelectric elements are excited at 1.67 MHz with $15.44 \mathrm{~V}_{\mathrm{PP}}$. The initial polystyrene concentration is $0.5 \%$ volume-to-volume ratio $(\mathrm{v} / \mathrm{v})$.

to it [Figs. 1(a) and 1(b)]. Upon excitation of the piezos with frequency $f$, the capillary starts to vibrate. When a suitable resonance of the fluid cavity inside the capillary is excited, particles migrate toward the center of the capillary, forming a thin particle line [Fig. 1(c)]. The force responsible for the particle migration toward the center of the capillary is called the acoustic radiation force (ARF). The ARF arises from the interactions between the incident acoustic field and the acoustic field scattered at a particle. For particles the radius of which is smaller than the viscous boundary layer thickness,

$$
\delta=\sqrt{\frac{\eta}{\pi \rho_{0} f}}
$$

with the density of the fluid at equilibrium $\rho_{0}$ and the dynamic viscosity $\eta$, viscous effects need to be considered when computing the ARF, since these could even lead to an inversion of the stable particle positions [20-22]. Here, we deal with particle radii $(r)$ just larger than the viscous boundary layer thickness, namely $r \sim 0.58 \mu \mathrm{m}>$ $\delta \sim 0.44 \mu \mathrm{m}$, while the acoustic wavelength $(\lambda)$ is much bigger than the particle radius, meaning that the inviscid ARF theory still offers a good approximation. The ARF is, 
therefore, given as [23]

$$
\mathbf{F}_{\mathrm{rad}}=-\nabla U,
$$

with the Gor'kov potential

$$
U=\frac{4}{3} \pi r^{3}\left(\frac{1}{2} \frac{f_{1}}{c_{0}^{2} \rho_{0}}\left\langle p_{1}^{2}\right\rangle-\frac{3}{4} \rho_{0} f_{2}\left\langle\mathbf{v}_{1} \cdot \mathbf{v}_{1}\right\rangle\right),
$$

where $p_{1}$ is the incident acoustic pressure field, $\mathbf{v}_{1}$ is the incident acoustic velocity field, $c_{0}$ is the fluid speed of sound, and $f_{1}$ and $f_{2}$ are the monopole and dipole scattering coefficients, respectively. Symbols in bold refer to vector quantities. $\langle\square\rangle$ denotes time averaging $\langle\square\rangle=$ $1 / T \int_{t_{1}}^{t_{1}+T} \square d t$, with any point in time $t_{1}$ and the period of oscillation $T=1 / f$.

Another nonlinear time-averaged effect that needs to be considered in our system is acoustic streaming. The force exerted on particles by acoustic streaming is the Stokes' drag $[24,25]$

$$
\mathbf{F}_{\mathrm{str}}=6 \pi \eta r\left(\mathbf{v}_{\mathrm{str}}-\mathbf{v}_{\mathrm{prt}}\right),
$$

where $\mathbf{v}_{\text {prt }}$ is the particle velocity and $\mathbf{v}_{\text {str }}$ is the streaming velocity. The latter can be determined using numerical $[10,20]$ and experimental methods [19], which allows for the consideration of arbitrary geometries. In our case, we employ the finite-element method (FEM) to compute the acoustic streaming field.

Due to the different scaling with the particle radius $r$ of the two forces, namely $\mathbf{F}_{\text {rad }}$ [scales with $r^{3}$, Eq. (3)] and $\mathbf{F}_{\text {str }}$ [scales with $r$, Eq. (4)], a critical radius $r_{c}$ exists at which the drag force from the streaming flow and the ARF are of equal magnitude. This critical particle radius is typically derived by balancing the two forces and solving for the radius. For a one-dimensional (1D) standing wave parallel to a planar wall (rectangular devices), the critical radius can be approximated by [15]

$$
r_{c}^{1 \mathrm{D}}=\sqrt{\frac{3 \psi}{2 \Phi}} \delta
$$

with the geometry-dependent factor $\psi=3 / 8$ [26] valid for rectangular cross sections and the acoustic contrast factor $\Phi$, which can be written as

$$
\Phi=\frac{1}{3} f_{1}+\frac{1}{2} f_{2}=\frac{1}{3}\left[\frac{5 \tilde{\rho}-2}{2 \tilde{\rho}+1}-\tilde{\kappa}\right],
$$

in which the relative compressibility $\tilde{\kappa}=\kappa_{p} / \kappa_{f}$ and the equilibrium density $\tilde{\rho}=\rho_{p} / \rho_{f}$ reflect the ratios between the particle $(\square)_{p}$ and fluid $(\square)_{f}$ properties. In the case of PS particles dispersed in water, the acoustic contrast factor is approximately $\Phi \approx 0.17$ and for copper particles dispersed in water, $\Phi \approx 0.75$. In commonly used rectangular acoustofluidic channels, with an excitation frequency of $f \sim 1.75 \mathrm{MHz}$, the critical radius is evaluated at around $r_{c}^{1 \mathrm{D}} \approx 0.37 \mu \mathrm{m}$ for a copper particle in water and around $r_{c}^{\mathrm{ID}} \approx 0.78 \mu \mathrm{m}$ for a PS particle in water. Our system, however, appears to have a lower critical radius, since we experimentally prove particle focusing with $r \sim 0.5 \mu \mathrm{m}$ for PS particles in water.

In the analytical approximation presented above, the maximum of the streaming velocity is compared with the maximum of the acoustic radiation force, these two not necessarily being the same spatial position, leading to rather approximate values. Therefore, we evaluate the forces numerically at each spatial position and compute the critical particle radius using the following formulation:

$$
r_{c}=\sqrt{\frac{6 \pi \eta\left\|\mathbf{v}_{\mathrm{str}}\right\|}{\frac{4}{3} \pi\left\|\nabla\left(\frac{1}{2} \frac{f_{1}}{c_{0}^{2} \rho_{0}}\left\langle p_{1}^{2}\right\rangle-\frac{3}{4} \rho_{0} f_{2}\left\langle\mathbf{v}_{1} \cdot \mathbf{v}_{1}\right\rangle\right)\right\|}} .
$$

The numerical investigations reveal a highly frequencydependent behavior with a minimal critical particle radius of $0.35 \mu \mathrm{m}$ for PS particles specifically for our geometry, exposing the strong influence of the channel geometry and frequency on the critical particle radius. However, even by our numerical investigations, the critical particle radius obtained by balancing the two force amplitudes is a very rough estimation, which cannot guarantee perfect particle focusing due to the spatially dependent radiation force and streaming. Hence, the exact critical particle radius always needs to be verified experimentally [19].

As mentioned beforehand, the acoustic effects inside the fluid cavity become significant when the system is close to resonance. The latter can be pinpointed by the analysis of the average acoustic energy density $\left(\bar{E}_{\mathrm{ac}}\right)$, which is given as [27]

$$
\bar{E}_{\mathrm{ac}}=\frac{1}{V} \int_{V}\left(\frac{1}{2} \rho_{0}\left\langle\mathbf{v}_{1} \cdot \mathbf{v}_{1}\right\rangle+\frac{1}{2} \kappa\left\langle p_{1}^{2}\right\rangle\right) d V
$$

where $\kappa$ is the compressibility of the fluid and $V$ is the volume.

\section{MATERIALS AND METHODS}

\section{A. Device fabrication}

Two piezoelectric elements $(10 \mathrm{~mm}$ length, $2 \mathrm{~mm}$ width, $1 \mathrm{~mm}$ thickness, Pz26, Meggitt Ferroperm, Denmark) are glued to the bottom and side of a round glass capillary $(0.5 \mathrm{~mm}$ inner diameter, $1 \mathrm{~mm}$ outer diameter, 76 mm length, 1B100-3, World Precision Instruments, Germany) using conductive epoxy (H20E, Epoxy Technology, Switzerland). Preliminary experiments show that the orientation of the piezoelectric elements with respect to one another can be chosen arbitrarily when attached to a 
round capillary. Instead of two piezos, a single longer element can also be utilized. However, we aim at avoiding the use of a bulky power amplifier by keeping the input power below the limit of the wave generator while increasing the focusing length. Copper cables $(0.15 \mathrm{~mm}$ diameter $)$ are attached to the piezo with conductive silver paste and glued to the capillary with instant glue to increase the mechanical stability.

For the particle-ejection experiments, glass nozzles are fabricated by pulling thin-walled borosilicate glass capillaries $(1 \mathrm{~mm}$ outer diameter, $0.75 \mathrm{~mm}$ inner diameter, TW100-4, World Precision Instruments, Germany) using a pipette puller (P-97, Sutter Instruments, USA) equipped with a $2.5 \times 2.5 \mathrm{~mm}$ platinum-iridium box filament. The nozzle size is tuned by varying the number of pulling cycles, which is achieved by changing the velocity of the puller bars at which the filament heating stops. Nozzle outer diameters in the range from 10 to $50 \mu \mathrm{m}$ are achieved by repetitive pulling cycles until the capillary separates into two identical nozzles. The tips of the capillaries (nozzles) are dipped into a fluorophilic polymer (Novec $^{\mathrm{TM}} 1700,3 \mathrm{M}^{\mathrm{TM}}$, Switzerland) to render it hydrophobic, enabling water ejection as a thin jet by avoiding wetting of the surface of the nozzles, which would eventually lead to droplet formation.

\section{B. Experimental setup}

The bulk-acoustic-wave (BAW) devices are based on the generation of ultrasonic standing waves in a fluid cavity. The waves are coupled into the devices by exciting the piezoelectric elements with an ac signal. The signals are generated via a wave generator (AFG-2225, GW INSTEK, Taiwan), using the maximal output of $10 \mathrm{~V}_{\mathrm{PP}}$. The impedance of the piezo varies with its excitation frequency. Since the piezo voltage depends on its impedance, it is verified using an oscilloscope (UTD2025CL, UniTrend Technology, China) leading to a piezo voltage of 15.44 $\mathrm{V}_{\mathrm{PP}}$ at $1.67 \mathrm{MHz}$ and $1.74 \mathrm{MHz}$. Through particle velocimetry analysis revealing a maximal particle velocity of approximately $100 \mu \mathrm{m} \mathrm{s}^{-1}$ and analytical approximations, we find an approximate value for the acoustic energy density of roughly $E_{\mathrm{ac}} \approx 10 \mathrm{~J} \mathrm{~m}^{-3}$, which is in the range of the values found in the literature [28]. However, since this value is only valid for a $1 \mathrm{D}$ plane wave and our system exhibits a rather complex 2D wave field, it should only be used as a rough order-of-magnitude approximation. Water flow inside the capillary is controlled by a syringe pump (neMESYS 290N, Cetoni, Germany). Two syringes are used for the particle-ejection experiments, one containing metal particles diluted in distilled water and stabilized with Tween 20 and the other containing solely distilled water. First, the capillary is filled with distilled water. Then, the metal-particle flow is slowly increased to achieve the desired concentration and flow rate. If the metal particles are directly inserted into the syringe, the nozzle clogs immediately due to unavoidable particle clumps. A self-built syringe mixer is utilized to increase the homogeneity of the ejection. The mixer consists of five copper coils linearly arranged along with the syringe, each controlled with a MOSFET (IRF540N, Infineon, Germany) and a microcontroller (Arduino Nano, Arduino, USA). A cylindrical iron piece is used as a stirrer by moving it up and down inside the syringe. This procedure is essential for copper particles, since they sediment much faster due to their higher density and then tend to form big clumps inside the syringe, impeding proper ejection.

For the optical visualization, a self-built microscope kit $\left(\right.$ Cerna $^{\circledR}$, Thorlabs, Germany) is utilized. A green light-emitting diode (LED) (M505L3, Thorlabs, Germany) excites the particles via a dichroic mirror (MD515, Thorlabs, Germany). Before entering the camera (UI-3180CP, IDS Imaging Development Systems GmbH, Germany), the light is filtered by an emission filter (MF535-22, Thorlabs, Germany). To visualize the side of our glass capillary, individual parts from the company Thorlabs are combined. A camera (UI-3180CP, IDS Imaging Development Systems $\mathrm{GmbH}$, Germany) is connected to a camera tube (WFA4100, Thorlabs, Germany), which is attached to a filter-cube housing (WFA2002, Thorlabs, Germany) with a filter cube (MDFM-MF2, Thorlabs, Germany) carrying a dichroic mirror (MD499, Thorlabs, Germany) and an emission filter (MF525-39, Thorlabs, Germany). The fluorescent filter sets are removed and two white LEDs are added as background lighting for the top and side view to improve the visibility if metal particles are flown through the capillary. A high-precision zoom housing (SM1ZM, Thorlabs, Germany), crucial for adjusting the focus, is mounted to the filter-cube housing with a combination of three parts (CSA1003, ER1-P4, LCP02/M, Thorlabs, Germany). the particle focusing is observed $2 \mathrm{~mm}$ away from the exit of the capillaries to ensure a maximal time for the particles to focus. Due to their long working distance, Mitutoyo objectives with a $5 \times$ magnification are connected to the microscopes.

For the device characterization, green fluorescent PS particles (microParticles $\mathrm{GmbH}$, Germany) with $5.19 \pm$ $0.14 \mu \mathrm{m}$ diameter (PS5) and $1.14 \pm 0.04 \mu \mathrm{m}$ diameter (PS1), and copper particles (Nanografi Nanotechnology, Turkey) with $1.16 \pm 0.61 \mu \mathrm{m}$ diameter $(\mathrm{Cu} 1)$ are used.The initial concentration is achieved by diluting the particles in distilled water. The local concentration after focusing is determined by deriving the ratio of the inner-diameter area and the elliptical-particle-stream area and multiplying it with the initial particle concentration.

\section{Numerical model}

A 2D numerical model of the cross section of the device (including piezo) is built in COMSOL Multiphysics version 
5.4. After studying the frequency response of the device, the displacement of the capillary as well as the Gor'kov potential and the streaming velocity close to the frequency exposing the lowest critical particle radius $f \approx 1.74 \mathrm{MHz}$ is analyzed. Further, the particle dynamics are assessed through the streaming velocity and the ARF, which is computed from the Gor'kov potential [Eq. (2)].

A user-controlled mesh with several mesh refinements is chosen, especially at the interfaces between different domains, to correctly incorporate streaming into our numerical model. For a more detailed mesh analysis, see Fig. S-1 in the Supplemental Material [29]. A frequencydomain study is used to solve (i) the Thermoviscous Acoustics interface applied to the water domain, (ii) the Solid Mechanics interface applied to the capillary, glue, and piezo domain, and (iii) the Electrostatics interface applied to the piezo domain. Further, a stationary study of the Creeping Flow interface applied to the water domain is carried out using the acoustic fields from the frequencydomain study in the source terms of the streaming equations. This study returns the streaming-velocity field as a direct result. Finally, a time-dependent study of the Particle Tracing for Fluid Flow interface is carried out by taking the acoustic and streaming-velocity fields into account. For a more detailed description of the numerical model, see Sec. SI-1 of the Supplemental Material [29].

\section{Determination of the particle line width}

A self-written MATLAB script is used to analyze the performance of the device by estimating the width of the focused particle line. The script takes a video as input and averages 20 frames to account for flow instabilities.
After adjusting the region of interest, a concentration profile is created by extracting all pixel brightness values in this region. The brightness values along the flow direction are summed up to receive the brightness distribution along the width of the channel. The brightness distribution is fitted with a Gaussian curve and the particle line width is determined with the fitted curve by measuring the width of the Gaussian fit after two standard deviations, i.e., when approximately $70 \%$ of the particles are within this region [see Fig. 2(a)]. The noise is significantly reduced by subtracting a reference image without any particles from the brightness distribution.

\section{RESULTS AND DISCUSSION}

\section{A. Experimental characterization of the device}

We experimentally investigate the performance of the acoustofluidic device. First, we vary the excitation frequency and choose the frequency that yields the thinnest particle line by visual inspection. A frequency between 1.67 and $1.78 \mathrm{MHz}$ results in the best particle focusing, which is close to the frequency predicted by our numerical model, despite its simplicity (2D geometry). To qualitatively examine the ability of our device to focus particles of different sizes and material properties, we analyze the line width of the focused particles by evaluating videos with a self-written MATLAB code [Sec. III D, Fig. 2(a)].

We measure the particle line width of PS particles $5.19 \pm 0.14 \mu \mathrm{m}$ (PS5) and $1.14 \pm 0.04 \mu \mathrm{m}$ (PS1) in diameter and copper particles $1.16 \pm 0.61 \mu \mathrm{m}(\mathrm{Cu} 1)$ in diameter to characterize the device performance. The particles are dispensed in water with a concentration of $0.5 \%$ $\mathrm{v} / \mathrm{v}$ (initial concentration) and pumped through the device (a)

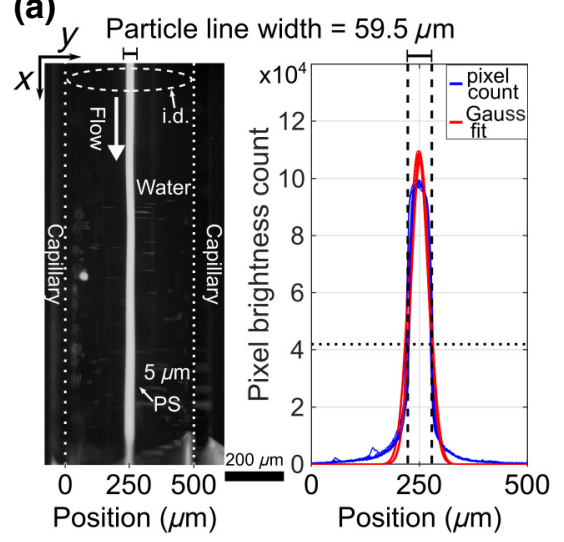

(b)

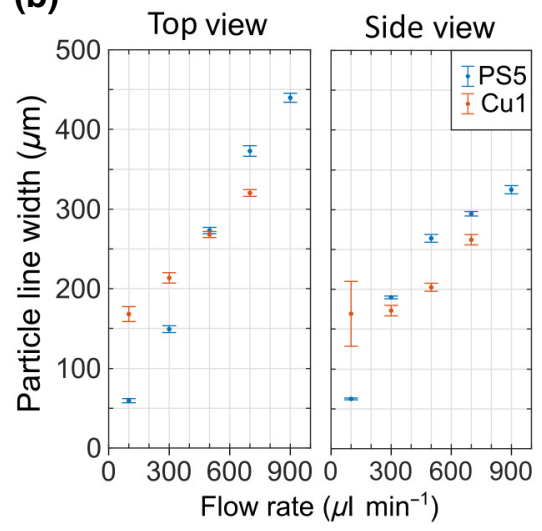

(c)

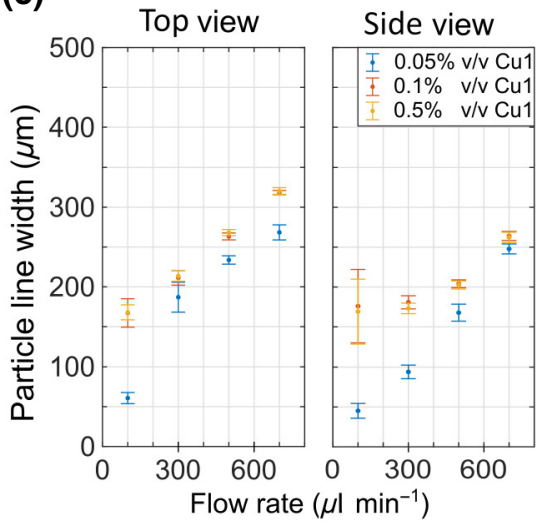

FIG. 2. The experimental device characterization. (a) An optical microscopy image of PS5 particles diluted in water with $0,5 \% \mathrm{v} / \mathrm{v}$, focused with an excitation frequency $f=1.74 \mathrm{MHz}$ and voltage $V=15.44 \mathrm{~V}_{\mathrm{PP}}$ at a flow rate of $Q=200 \mu 1 \mathrm{~min}^{-1}$. The corresponding analysis of the particle line width with the MATLAB code described in Sec. III D is shown on the right-hand side. (b) The particle line width of PS5 and Cu1 particles diluted in water with a concentration of $0.5 \% \mathrm{v} / \mathrm{v}$. The line width increases with an increasing flow rate. The line width of PS5 and Cu1 particles are comparable despite the difference in size due to the different acoustic contrast factor. (c) The particle line width for different initial concentrations of Cul. The line width increases with an increasing initial concentration and flow rate. 
(a)

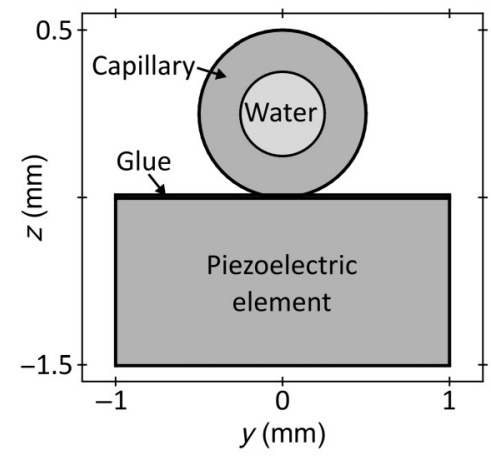

(d)

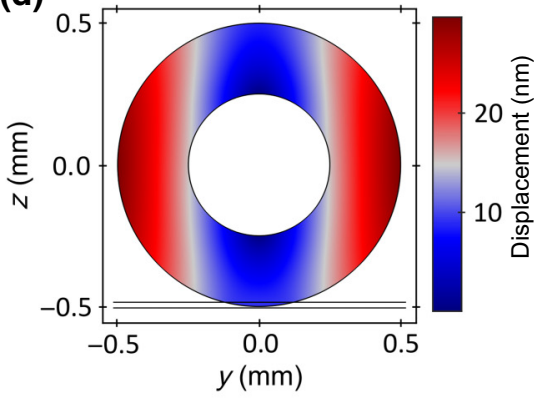

(b)

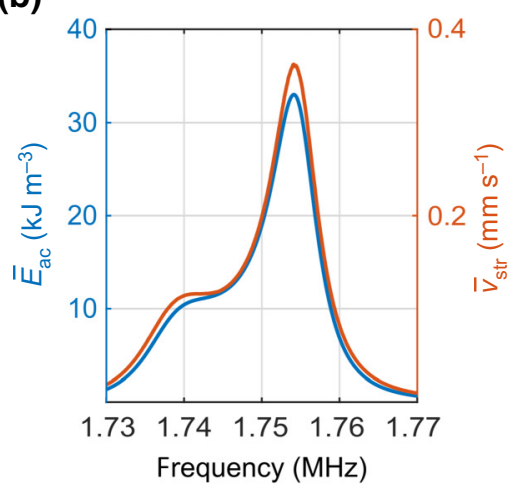

(e)

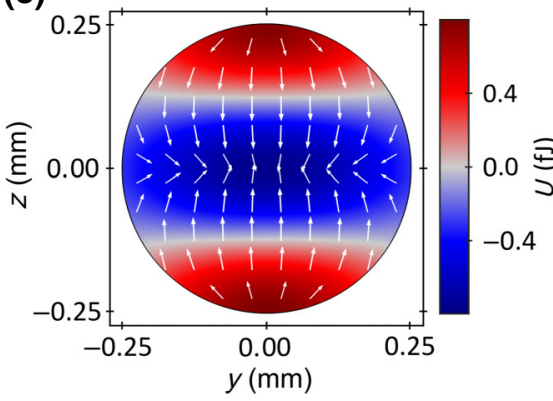

(c)

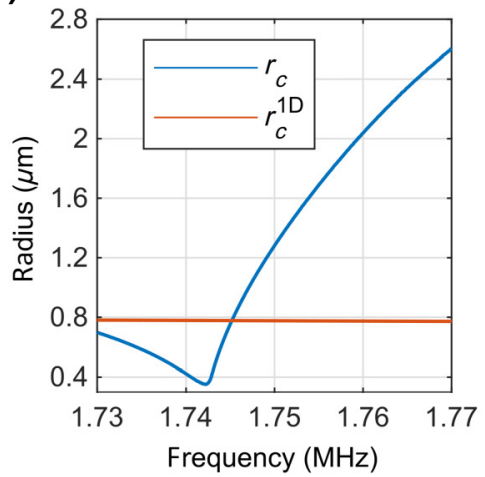

(f)

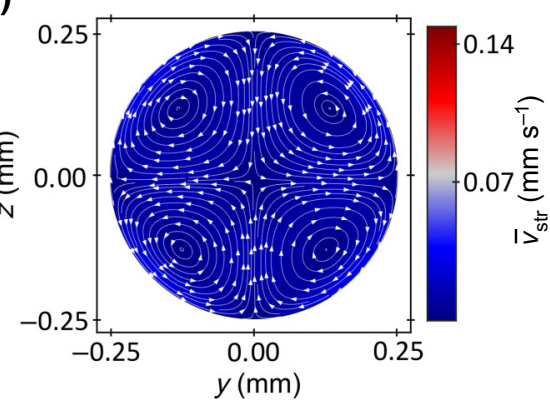

FIG. 3. The numerical analysis of the acoustofludic device. (a) The numerical model of the round capillary with a piezo attached to the bottom via a glue layer with a thickness of $20 \mu \mathrm{m}$. (b),(c) The frequency sweep from 1.73 to $1.77 \mathrm{MHz}$ with steps of $0.1 \mathrm{kHz}$. (b) At $f=1754.1 \mathrm{kHz}$, a maximum in $\bar{v}_{\text {str }}$ and $\bar{E}_{\text {ac }}$ can be found. (c) A comparison of the analytically $\left[r_{c}^{1 \mathrm{D}}\right.$ Eq. (5)] and numerically $\left[r_{c}\right.$ Eq. (7)] derived critical particle radius for PS particles revealing a local minimum at $f=1742.2 \mathrm{kHz}$ that is not apparent in (b). (d)-(f) The capillary average-displacement magnitude (d), the Gor'kov potential with white arrows (logarithmic scaling) as the ARF (e), and the streaming patterns (f) at $f=1742.2 \mathrm{kHz}$ indicate that particles are pushed toward the center of the capillary.

at flow rates in a range of $5 \mu 1 \mathrm{~min}^{-1}\left(0.11 \mathrm{~mm} \mathrm{~s}^{-1}\right.$ average velocity) to $900 \mu 1 \mathrm{~min}^{-1}\left(19.14 \mathrm{~mm} \mathrm{~s}^{-1}\right.$ average velocity). At approximately $15 \mathrm{~V}_{\mathrm{PP}}\left(E_{\mathrm{ac}} \approx 10\right)$, the acoustic radiation force in the flow direction is already strong enough to trap particles in the center of the device, in the focus area of the piezos, below flow rates of $100 \mu 1 \mathrm{~min}^{-1}$ (2.13 $\mathrm{mm} \mathrm{s}^{-1}$ average velocity). Therefore, we are not able to analyze the line width below a flow rate of $100 \mu 1 \mathrm{~min}^{-1}$ for the PS5 and Cu1 particles. The PS1 particles do not get trapped even at low flow rates, since the acoustic radiation force is approximately $125 \times$ lower [Eq. (2)]. Despite the small size, which would usually impede a focus of these particles using acoustic effects, we are able to focus the PS1 particles at a flow rate of $5 \mu 1 \mathrm{~min}^{-1}$ to a line of width $80.6 \pm 6.9 \mu \mathrm{m}$ (top view) and height $65.0 \pm 3.9 \mu \mathrm{m}$ (side view) - see Video 1 in the Supplemental Material [29]. The ability to focus such small particles might be due to a minimum critical particle radius specific to our system, discovered by the numerical analysis [Fig. 3(c)]. At double the flow rate $\left(10 \mu 1 \mathrm{~min}^{-1}\right)$, the line width of the PS1 particles increases drastically to $401.5 \pm 19.9 \mu \mathrm{m}$ (top view) and $277.6 \pm 18.2 \mu \mathrm{m}$ (side view). Above the flow rate of $10 \mu 1 \mathrm{~min}^{-1}$, particle focusing is no longer observable due to the too short focusing time.
Following the experimental focusing of PS1 particles, we insert the PS5 particles into the system. At a flow rate of $100 \mu \mathrm{min}^{-1}$, the PS5 particles can be focused down to a line of width of $62.6 \pm 1.1 \mu \mathrm{m}$ (top view) and height $59.5 \pm 2.6 \mu \mathrm{m}$ (side view), corresponding to a local concentration of $34 \% \mathrm{v} / \mathrm{v}$. With a linear increase of the flow rate, the line width also increases linearly, as the time available to manipulate the particles inside the device is reduced. Even at a flow rate of $900 \mu 1 \mathrm{~min}^{-1}$, we are able to focus the PS5 particles down to a line of width $439.6 \pm 5.7 \mu \mathrm{m}$ (top view) and height $325.2 \pm 5.2 \mu \mathrm{m}$ (side view).

Next, we insert Cul particles into the device. Due to the significantly larger density and the compressibility difference compared to water - an approximately $4.4 \times$ bigger acoustic contrast factor [Eq. (6)] - the copper particles experience a larger ARF compared to the PS particles of the same size. This leads to similar particle line widths for the $\mathrm{Cu} 1$ and PS5 particles despite their difference in size. At a flow rate of $100 \mu \mathrm{min}^{-1}$, the copper particles can be focused down to a line of width $168.0 \pm 9.4 \mu \mathrm{m}$ (top view) and height $169.1 \pm 40.8 \mu \mathrm{m}$ (side view), corresponding to a local concentration of $4.4 \% \mathrm{v} / \mathrm{v}$ - see Video 2 in the Supplemental Material [29]. Even at high flow rates of 
$700 \mu 1 \mathrm{~min}^{-1}$, we achieve a line of width $320.4 \pm 4.2 \mu \mathrm{m}$ (top view) and height $262.3 \pm 6.4 \mu \mathrm{m}$ (side view). All particle line widths for the tested flow rates and particle materials are given in Fig. 2(b). Interestingly, the particle line width of PS5 particles is lower than that of $\mathrm{Cu} 1$ particles at flow rates below $600 \mu 1 \mathrm{~min}^{-1}$ but higher at flow rates above this value. The basic principles causing this phenomenon are as yet unknown.

Finally, we evaluate the influence of the initial copperparticle concentration on the particle line width. We test three different concentrations $(0.05 \% \mathrm{v} / \mathrm{v}, 0.1 \% \mathrm{v} / \mathrm{v}$, and $0.5 \% \mathrm{v} / \mathrm{v}$ ) and find an increase in particle line width for an increasing flow rate regardless of the concentration [Fig. 2(c)]. Additionally, the particle line width increases for increasing initial particle concentrations, which could be attributed to the maximal particle packing density and interparticle effects. Finally, especially at high flow rates, the concentration only has a negligible influence on the particle line width. Therefore, one can assume that particleparticle interactions play only a minor role in comparison to other relevant forces at flow rates above $500 \mu 1 \mathrm{~min}^{-1}$.

For an initial concentration of $0.05 \% \mathrm{v} / \mathrm{v}$, we are able to focus the $\mathrm{Cu} 1$ particles into a line of width $60.8 \pm$ $7.0 \mu \mathrm{m}$ (top view) and height $45.2 \pm 9.3 \mu \mathrm{m}$ (side view) at a flow rate of $100 \mu 1 \mathrm{~min}^{-1}$, corresponding to a local concentration of $4.5 \% \mathrm{v} / \mathrm{v}$.

\section{B. Numerical analysis of the particle manipulation}

We utilize the numerical model described in Sec. III C to study the underlying physical phenomena that lead to $2 \mathrm{D}$ particle focusing. A frequency sweep from 1 to $2.5 \mathrm{MHz}$ $(0.1 \mathrm{kHz}$ step) reveals a strong resonance of the system at $1754.1 \mathrm{kHz}$, indicated by a peak in the average acoustic energy density $\left(\bar{E}_{\mathrm{ac}}\right)$ and the average streaming velocity $\left(\bar{v}_{\text {str }}\right)$ [see Fig. 3(b)]. Even though the large acoustic energy density and the Gor'kov potential are favorable for particle focusing in the center of the capillary, the strong acoustic streaming disturbs the ARF-driven focusing of small particles. We confirm the undesirable influence of the acoustic streaming by particle-trajectory simulations, where the copper particles $(r=0.5 \mu \mathrm{m})$ end up being carried around with the streaming vortices, which contradicts the experimentally observed results. Therefore, we numerically evaluate the critical particle radius by balancing the maximal streaming-velocity magnitude and the maximal ARF magnitude in the computational domain using Eq. (7) and compare it to the analytical approximation [Eq. (5)]. In contrast to the analytical result, the numerical evaluation reveals a local minimum of the critical particle radius at $f=1742.2 \mathrm{kHz}$, explaining our capability to focus the PS particles with $r=0.5 \mu \mathrm{m}$ radius [see Fig. 3(c)].

At $f=1742.2 \mathrm{kHz}$, the capillary exhibits displacements in the nanometer range, as shown in Fig. 3(d). The Gor'kov potential in Fig. 3(e) with maxima at the top and bottom of the capillary and a minimum in the center indicates the attraction of particles toward the $z=0$ plane in the glass capillary. The acoustic radiation force arrows in Fig. 3(e) point toward the capillary center, which confirms the attraction of the particles toward the center of the capillary. The acoustic streaming field consists of four vortices that have a joint point of zero velocity close to the center of the capillary 3(f), an observation that is beneficial for particle focusing.

The streaming velocity at $f=1742.2 \mathrm{kHz}$ is low compared to the streaming velocity at the nearby frequencies. This could be attributed to the transition of the streaming pattern, which appears to weaken the overall acoustic streaming (see Fig. 4). Another explanation for the reduced acoustic streaming in our system could be due to the curved shape of our channel walls, which is optimal as suggested by Bach et al. [18]. In their publication, Bach et al. explain that the acoustic streaming velocity along the boundaries is proportional to the gradient of the acoustic pressure
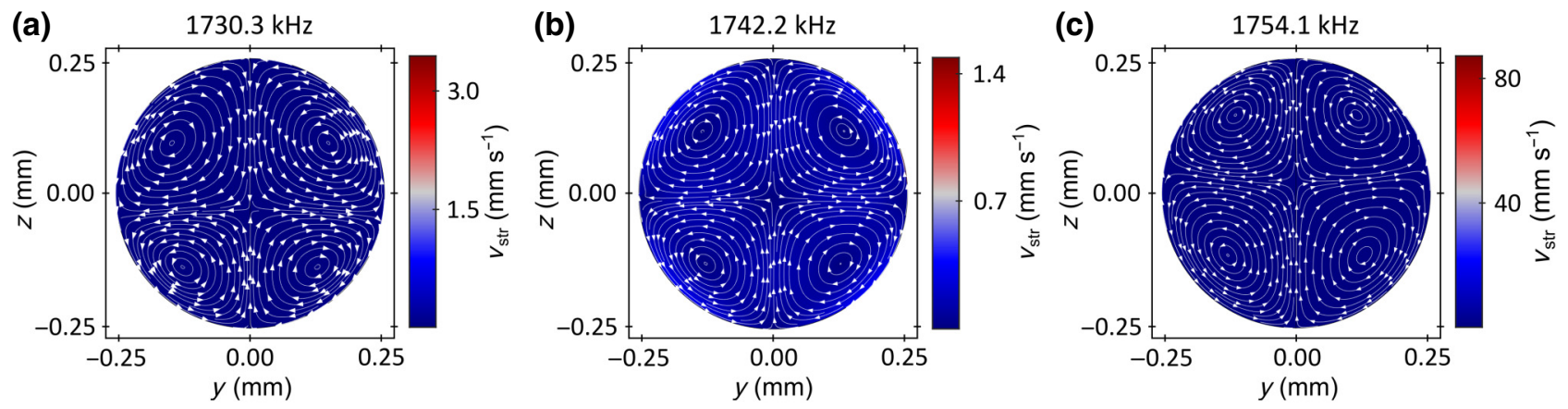

FIG. 4. The numerical streaming patterns. The streaming patterns at (a) $f=1730.3 \mathrm{kHz}$, (b) $f=1742.2 \mathrm{kHz}$, and (c) $f=1754.1 \mathrm{kHz}$. The color indicates the amplitude of the streaming velocity, while the arrows on the streamlines indicate the flow direction. The results are given for $20 \mathrm{~V}_{\mathrm{PP}}$ excitation of the piezo. The maximal streaming velocity can be found within the viscous boundary layer, which is not visible in the images due to the small size. (b) At the local streaming minimum, four streaming vortices form that are approximately equal in size and strength. If the frequency is slightly decreased (a) or increased (c), the streaming amplitude increases significantly and the upper (a) or lower (c) vortex pair starts to dominate. 
along the boundaries. Since curved boundaries exhibit a rather linear acoustic pressure profile along the boundaries, the acoustic streaming is suppressed in comparison to rectangular cross sections.

Using particle-trajectory simulations, we confirm that the influence of the acoustic streaming at $f=1742.2 \mathrm{kHz}$ is sufficiently reduced to yield the focusing of $r=2.5 \mu \mathrm{m}$ PS and $r=0.5 \mu \mathrm{m}$ copper particles in the center of the capillary (see Fig. S-2 in the Supplemental Material [29]). Considering the 3D acoustic field in the experiments and the related variability in the ARF and the streaming-velocity field, the simulations fit our experimental results nicely.

In addition to the reduced acoustic streaming, acoustic interparticle effects could contribute to our ability to focus particles in the low-micrometer range. At high concentrations, acoustic rescattering amongst particles leads to forces that can significantly influence the focusing capabilities [30]. Since, in our case, the viscous boundary layer thickness around the particles is close to their respective radii, theoretical approaches that consider the fluid viscosity would need to be employed [31]. However, the implementation of such theoretical approaches is challenging and requires advanced numerical models that are beyond the scope of this paper.

\section{Focused metal-particle ejection}

For the precise ejection of focused metal particles - as, e.g., needed for metal 3D printing - a tapered round capillary is connected to the already characterized round glass capillary (particle-focusing capillary) with a combination of silicone and heat-shrink tubing (see Fig. S-4 in the Supplemental Material [29]). We test various nozzle diameters ranging from 10 to $50 \mu \mathrm{m}$. Nozzles with a diameter below $20 \mu \mathrm{m}$ clog even at low initial $\mathrm{Cu} 1$ concentrations of $0.05 \% \mathrm{v} / \mathrm{v}$, regardless of prefocusing in the particlefocusing capillary. As can be seen in Fig. 5(b), nozzles with $25 \mu \mathrm{m}$ diameter also clog within $10 \mathrm{~s}$ when $\mathrm{Cu} 1$ particles with an initial concentration of $2 \% \mathrm{v} / \mathrm{v}$ are flushed through the system at a flow rate of $200 \mu 1 \mathrm{~min}^{-1}\left(4.25 \mathrm{~mm} \mathrm{~s}^{-1}\right.$ average velocity) and the prefocusing system is turned off (see Video 3 in the Supplemental Material [29]). However, as shown in Fig. 5(c), when the ultrasound of our particle-focusing capillary is switched on using an excitation frequency of $1.72 \mathrm{MHz}$, we are able to eject $\mathrm{Cu} 1$ particles with an initial concentration of $2 \% \mathrm{v} / \mathrm{v}$ at a flow rate of $200 \mu 1 \mathrm{~min}^{-1}$ (4.25 $\mathrm{mm} \mathrm{s}^{-1}$ average velocity) in a jetting mode (see Video 4 in the Supplemental Material [29]). By utilizing our prefocusing system, we are able to prevent the clogging of the $25-\mu \mathrm{m}$ diameter nozzle for $1 \mathrm{~mL}$ of sample solution. The prefocused metal particles are squeezed together in the nozzle, resulting in a particle stream of below approximately $4 \mu \mathrm{m}$ in diameter, and are ejected with an average velocity of
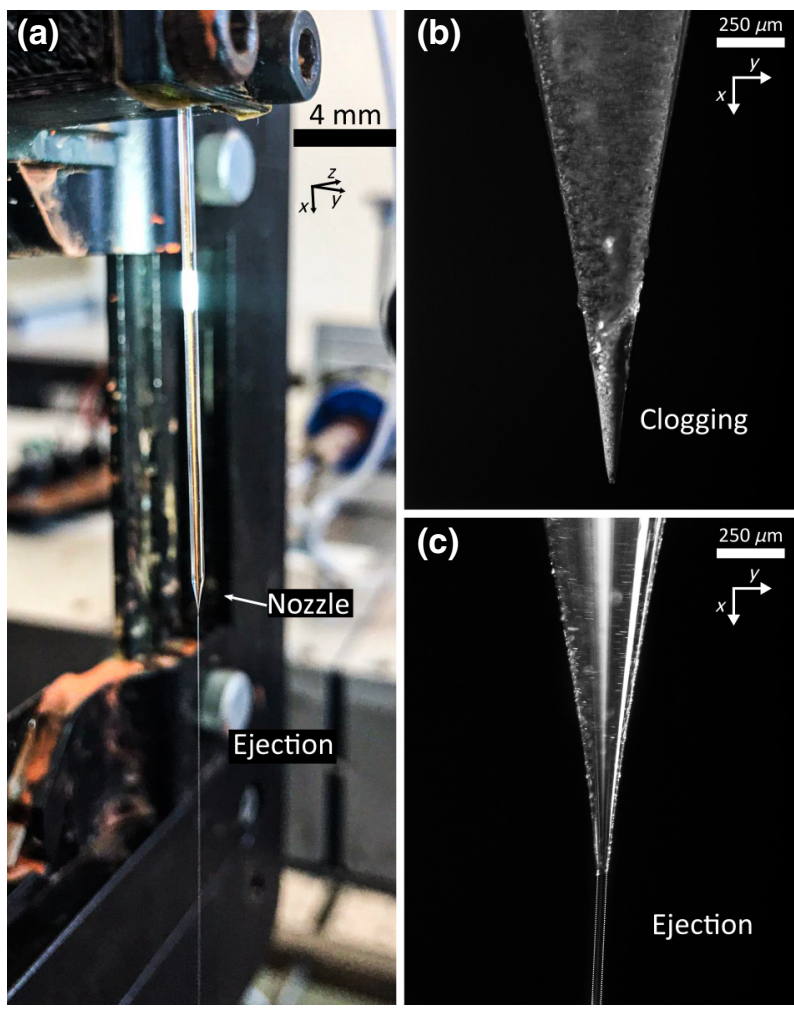

FIG. 5. The ejection of focused metal particles through a tapered round capillary. (a) A photograph of a tapered round glass capillary with a nozzle diameter of $25 \mu \mathrm{m}$. Water with a flow rate of $200 \mu \mathrm{min}^{-1}$ is flushed through the system, leading to ejection in the jetting mode. (b) An optical microscopy image of a clogged tapered capillary with a nozzle diameter of $25 \mu \mathrm{m}$. $\mathrm{Cu} 1$ particles with an initial concentration of $2 \% \mathrm{v} / \mathrm{v}$ are pumped through the system at a flow rate of $200 \mu 1 \mathrm{~min}^{-1}$ without prefocusing, leading to clogging of the nozzle within seconds. (c) An optical microscopy image of a tapered capillary with a nozzle diameter of $25 \mu \mathrm{m}$ while ejecting particles in the jetting mode. $\mathrm{Cu} 1$ particles with an initial concentration of $2 \% \mathrm{v} / \mathrm{v}$ are flown through the system at a flow rate of $200 \mu \mathrm{min}^{-1}$. The particles are prefocused within our focusing capillary, which is excited at a frequency of $f=1.72 \mathrm{MHz}$.

approximately $1.7 \mathrm{~m} \mathrm{~s}^{-1}$. We test this procedure repeatedly without exchanging the nozzle; hence we believe that much larger volumes can be ejected without clogging the nozzle when utilizing our system.

With the combination of our particle-focusing capillary and a tapered round capillary, we are able to prove that 2D particle focusing within a round glass capillary can be utilized to impede the clogging of a thin nozzle.

\section{CONCLUSION AND OUTLOOK}

In this work, we show 2D focusing of micron-sized particles and their stable ejection through a nozzle that is about 20 times larger. 
We carry out numerical investigations to improve our understanding of 2D particle focusing inside round glass capillaries. We find an excitation frequency at which the influence of the acoustic streaming is weak compared to the acoustic radiation force and we compute a critical particle radius specific to our system by numerical investigations, explaining our ability to focus particles sized close to the theoretical minimum. The role of acoustic and hydrodynamic interparticle effects remains to be resolved and can be the subject of future research, in which we combine the methods of Sepehrirahnama et al. [31] with a time-stepping algorithm and account for hydrodynamic interactions $[32,33]$.

Through experimental evaluations, we are able to determine the performance of our device. At a flow rate of $5 \mu 1 \mathrm{~min}^{-1}$, we are able to focus polystyrene particles with a diameter of $1 \mu \mathrm{m}$ into a line of width $80.6 \pm 6.9 \mu \mathrm{m}$ and height $65.0 \pm 3.9 \mu \mathrm{m}$. Additionally, at a 20 times faster flow speed, we focus $5 \times$ bigger polystyrene particles into a line of similar size. Copper particles with a diameter of $1 \mu \mathrm{m}$ are observed to behave similarly to polystyrene particles that are 5 times bigger due to the significantly higher acoustic contrast.

Finally, we demonstrate the ejection of a $2 \% \mathrm{v} / \mathrm{v}$ concentration of $1 \mu \mathrm{m}$ copper particles through a nozzle of diameter $25 \mu \mathrm{m}$, which is unattainable without prior acoustic focusing. The device performance could be further improved by using more or longer piezoelectric elements, leading to a more extended focusing region that demands higher input power. Further, if needed, the contact area of the piezoelectric elements could be enhanced by special holders or curved surfaces of the piezoelectric elements, improving the mechanical coupling.

With our approach, the reliability of systems that rely on particle ejection through a small nozzle and are subject to abrasion, such as water-jet cutting, could be significantly increased. Therefore, our investigations are expected to be relevant for a wide variety of industrial applications, including water-jet cutting and metal $3 \mathrm{D}$ printing. In the case of metal 3D printing, we propose a combination of the described metal-particle focusing with electrohydrodynamic ejection, microwave drying, and laser melting to improve the resolution of state-of-the-art systems [34].

The data that support the findings of this study are available within the paper and its Supplemental Material [29,35-39]. The simulation model is available from the corresponding author upon request.

\section{ACKNOWLEDGMENTS}

We would like to express our gratitude for funding by ETH Zurich. This work was funded by the program of the Strategic Focus Area Advanced Manufacturing (SFAAM), a strategic initiative of the ETH Board. The project can be found under the name "Powder Focusing" [34]. We thank Dr. Nino Läubli for his valuable feedback and inputs.

[1] T. Laurell and A. Lenshof, eds. Microscale Acoustofluidics. The Royal Society of Chemistry (2015).

[2] M. W. Adem Ozcelik, J. Rufo, Z. Wang, R. Fang, and T. Jun Huang, Acoustofluidic separation of cells and particles, Microsystems Nanoeng. 5, 32 (2019).

[3] Y. Xia and G. M. Whitesides, Soft lithography, Annu. Rev. Mater. Sci. 28, 153 (1998).

[4] M. S. Gerlt, N. F. Läubli, M. Manser, B. J. Nelson, and J. Dual, Reduced etch lag and high aspect ratios by deep reactive ion etching (DRIE), Micromachines 12, 542 (2021).

[5] M. Wiklund, S. Nilsson, and H. M. Hertz, Ultrasonic trapping in capillaries for trace-amount biomedical analysis, J. Appl. Phys. 90, 421 (2001).

[6] I. Gralinski, S. Raymond, T. Alan, and A. Neild, Continuous flow ultrasonic particle trapping in a glass capillary, J. Appl. Phys. 115, 054505 (2014).

[7] B. Hammarström, T. Laurell, and J. Nilsson, Seed particleenabled acoustic trapping of bacteria and nanoparticles in continuous flow systems, Lab Chip 12, 4296 (2012).

[8] Z. Mao, P. Li, M. Wu, H. Bachman, N. Mesyngier, X. Guo, S. Liu, F. Costanzo, and T. J. Huang, Enriching nanoparticles via acoustofluidics, ACS Nano 11, 603 (2017).

[9] J. Lei, F. Cheng, K. Li, and Z. Guo, Two-dimensional concentration of microparticles using bulk acoustomicrofluidics, Appl. Phys. Lett. 116, 033104 (2020).

[10] J. Lei, F. Cheng, and K. Li, Numerical simulation of boundary-driven acoustic streaming in microfluidic channels with circular cross-sections, Micromachines 11, 240 (2020).

[11] I/ Gralinski, T. Alan, and A. Neild, Non-contact acoustic trapping in circular cross-section glass capillaries: A numerical study, J. Acoust. Soc. Am. 132, 2978 (2012).

[12] M. W. H. Ley and H. Bruus, Three-Dimensional Numerical Modeling of Acoustic Trapping in Glass Capillaries, Phys. Rev. Appl. 8, 024020 (2017).

[13] G. Goddard and G. Kaduchak, Ultrasonic particle concentration in a line-driven cylindrical tube, J. Acoust. Soc. Am. 117, 3440 (2005).

[14] G. Goddard, J. C. Martin, S. W. Graves, and G. Kaduchak, Ultrasonic particle-concentration for sheathless focusing of particles for analysis in a flow cytometer, Cytometry Part A 69A, 66 (2006).

[15] H. Bruus, Acoustofluidics 10: Scaling laws in acoustophoresis, Lab Chip 12, 1578 (2012).

[16] M. Hoyos and A. Castro, Controlling the acoustic streaming by pulsed ultrasounds, Ultrasonics $\mathbf{5 3}, 70$ (2013).

[17] J. T. Karlsen, W. Qiu, P. Augustsson, and H. Bruus, Acoustic Streaming and its Suppression in Inhomogeneous Fluids, Phys. Rev. Lett. 120, 00 (2018).

[18] J. S. Bach and H. Bruus, Suppression of Acoustic Streaming in Shape-Optimized Channels, Phys. Rev. Lett. 124, 214501 (2020).

[19] W. Qiu, H. Bruus, and P. Augustsson, Particle-sizedependent acoustophoretic motion and depletion of microand nano-particles at long timescales, Phys. Rev. E 102, 013108 (2020). 
[20] T. Baasch, A. Pavlic, and J. Dual, Acoustic radiation force acting on a heavy particle in a standing wave can be dominated by the acoustic microstreaming, Phys. Rev. E 100, 061102 (2019).

[21] A. A. Doinikov, Acoustic radiation pressure on a rigid sphere in a viscous fluid, Proc. Royal Soc. London. Ser. A: Math. Phys. Sci. 447, 447 (1994).

[22] J. T. Karlsen and H. Bruus, Forces acting on a small particle in an acoustical field in a thermoviscous fluid, Phys. Rev. E 92, 043010 (2015).

[23] L. Gor'kov, On the forces acting on a small particle in an acoustical field in an ideal fluid, Sov. Phys. Dokl. 6, 773 (1962).

[24] W. L. M. Nyborg, in Physical Acoustics, vol. 2, p. 265. Elsevier (1965).

[25] J. Lighthill, Acoustic streaming, J. Sound Vib. 61, 391 (1978).

[26] P. B. Muller, R. Barnkob, M. J. H. Jensen, and H. Bruus, A numerical study of microparticle acoustophoresis driven by acoustic radiation forces and streaming-induced drag forces, Lab Chip 12, 4617 (2012).

[27] A. D. Pierce, Acoustics: An Introduction to its Physical Principles and Applications. Springer International Publishing, Woodbury, 3rd ed. (1991).

[28] H. Bruus, Acoustofluidics 7: The acoustic radiation force on small particles, Lab Chip 12, 1014 (2012).

[29] See the Supplemental Material at http://link.aps.org/supple mental/10.1103/PhysRevApplied.17.014043 for a mesh study, simulated particle trajectories, and a detailed description of the numerical model.

[30] G. T. Silva and H. Bruus, Acoustic interaction forces between small particles in an ideal fluid, Phys. Rev. E 90, 063007 (2014).

[31] S. Sepehrirahnama, F. S. Chau, and K.-M. Lim, Effects of viscosity and acoustic streaming on the interparticle radiation force between rigid spheres in a standing wave, Phys. Rev. E 93, (2016).

[32] T. Baasch, I. Leibacher, and J. Dual, Multibody dynamics in acoustophoresis, J. Acoust. Soc. Am. 141, 1664 (2017).

[33] J. F. Brady, R. J. Phillips, J. C. Lester, and G. Bossis, Dynamic simulation of hydrodynamically interacting suspensions, J. Fluid Mech. 195, 257 (1988).

[34] SFA. Powder focusing. https://www.sfa-am.ch/powderfocusing.html, accessed: 2021-08-31.

[35] V. A. Del Grosso and C. W. Mader, Speed of sound in pure water, J. Acoust. Soc. Am. 52, 1442 (1972).

[36] A. R. Selfridge, Approximate material properties in isotropic materials, IEEE Trans. Son. Ultrason. 32, 381 (1985).

[37] Meggitt Ferroperm. Properties of Pz26. https://www.meggi ttferroperm.com, accessed: 2021-05-21.

[38] Epotek. Properties of H20E. https://www.epotek.com/, accessed: 2021-05-21.

[39] H. Bruus, Theoretical Microfluidics Vol. 18 (Oxford University Press, Oxford, 2008). 